\title{
Les prénoms révolutionnaires Bibliographie
}

\section{Raphaël Bange, Serge Bianchi et Pierre-Henri Billy}

\section{(2) OpenEdition}

\section{Journals}

\section{Édition électronique}

URL : https://journals.openedition.org/ahrf/1837

DOI : $10.4000 /$ ahrf. 1837

ISSN : $1952-403 X$

Éditeur :

Armand Colin, Société des études robespierristes

\section{Édition imprimée}

Date de publication : 1 décembre 2000

Pagination : 139-151

ISSN : 0003-4436

\section{Référence électronique}

Raphaël Bange, Serge Bianchi et Pierre-Henri Billy, «Les prénoms révolutionnaires Bibliographie »,

Annales historiques de la Révolution française [En ligne], 322 | octobre-décembre 2000, mis en ligne le 22 mars 2006, consulté le 23 avril 2022. URL : http://journals.openedition.org/ahrf/1837 ; DOI : https:// doi.org/10.4000/ahrf.1837

Ce document a été généré automatiquement le 23 avril 2022

Tous droits réservés 


\title{
Les prénoms révolutionnaires Bibliographie
}

\author{
Raphaël Bange, Serge Bianchi et Pierre-Henri Billy
}

Généralités

1 A. Aulard, «Les prénoms et la loi », Rev. française, LXIV, 67-69.

2 R. Bange, «Jean ou Pierre Marie Le nombre de prénoms considéré comme un indicateur des convictions religieuses », Mélanges Michel Vovelle, Paris, 1997, 75-86.

- « Les prénoms de l'anII. Quand nos ancêtres appelaient leurs enfants Brutus Vendéen et Marie Ça ira ", La France généalogique, 193, 1996, 22-25.

Y. et A. Benoist, « Les prénoms des Conventionnels », Du Passé au présent, 1989, 32-46.

4 S. Bianchi, La révolution culturelle de l'an II, Paris, 1982.

5 P.-H. Billy, compte rendu de N. Hörsch, Republikanische Personennamen. Eine anthroponymische Studie zur Französischen Revolution, Tübingen, 1994, in Rev. de Linguistique romane, LX, 1996, 593-596.

- «L'état civil des enfants inconnus ou abandonnés en France (1793-anIV) », Actes du colloque IV, Patrom (Dijon, 24-26 septembre 1990), Tübingen, 1992, 134-145.

- « Le TitreIII du décret du 20 septembre 1792, ses modifications et leur application en France jusqu'au Directoire des naissances ", à paraître dans Akten des 18. Internationalen Kongresses für Namenforschung (Trier, 12-17 April 1993), Tübingen, 2000.

6 E. Biré, « La Révolution et l'enfance », Rev. de la Révolution, I, 1883, 64-91.

7 J. Bouineau, Les toges du pouvoir (1789-1799) ou la révolution de droit antique, Toulouse, 1986.

8 J.-A. Carney, Mémoire sur les noms à substituer aux noms de baptême, ou projet de prénoms étrangers à tout culte, et très diversifiés, Montpellier, an III.

9 G. Cholvy, « Adhésion ou refus du changement les prénoms de l'an II », Bull. du Centre d'Hist. contemporaine du Languedoc méditerranéen-Roussillon, 1988, 28-31.

10 P. Cordonnier, "Une origine possible des prénoms civiques", Annales hist. de la Révolution française, 150, 1958, 70-72. 

13-14, 1989, 43-75.

-Die republikanischen Frauennamen zur Zeit der französischen Revolution, Thèse, Heidelberg, 1984.

-Republikanische Personennamen. Eine anthroponymische Studie zur Französischen Revolution, Tübingen, 1994.

\section{LXXIV, 1921, 318-339.}

-Manuel des prénoms, Paris, 1922.

- « Les prénoms de l'an II », Révolution française, LXV, 1913, 496-525 ; LXVI, 1914, 15-31.

-Onomastique et Révolution tradition et changement. Noms de rues, noms de lieux, prénoms, in Nouvelle Rev. d'Onomastique, 13-14, 1989, 3-156. 

Cercle généal. de Saintonge, 6, 1989, 14-15.

Auvergne I, 1987. 1986. LXXVIII, 1930, 483 ; LXXXII, 1935, 214. d'Alsace, 47, 1979, 467-470. 1983, 42-45.

Angoumois, $c f$. AunisAnjou, Maine et Touraine 719-735.

Artois, $c f$. PicardieAunis, Saintonge et Angoumois 9, 1984, 11-12. Bordeaux-III, 1988.

A. Bande, Déchristianisation et résistance populaire en Alsace pendant l'an II, Maîtrise, Paris-

E. Bernardin, Strasbourg et l'institution de l'état civil laïc, 1792-1798, Maîtrise, Strasbourg,

J. Joachim, «Prénoms révolutionnaires », Rev. d'Alsace, LXXVI, 1929, 591, 831-832 ;

M. Kubler, « Des prénoms révolutionnaires... à Saint Napoléon », Annuaire des Amis de la bibliothèque humaniste de Sélestat, 36, 1986, 151-187.

J.-M. Le Minor, «Prénoms révolutionnaires dans le Bas-Rhin », Bull. du Cercle généal.

C. Oberreiner, « Prénoms révolutionnaires », Rev. d'Alsace, LXXIX, 1932, 388.

A. Saira, «Baptêmes civiques pendant la révolution », Rev. d'Alsace, LXXIX, 1932, 178.

- « Prénoms révolutionnaires », Rev. d'Alsace, LXXVIII, 1931, 558-559.

P. Stroh, « Deux baptêmes «patriotiques » à Wissembourg en 1790 », L'Outre-Forêt, 44,

Affiches d'Angers ou Moniteur du département de Maine et Loire, Angers, an II.

Docteur Gaëtan, «Les vocables révolutionnaires », Révolution française, XIV, 1888,

J. L'hermitte, "Pseudonymes et surnoms de chouans signalés ou poursuivis dans la Sarthe de l'an III à l'an VIII ", Révolution dans la Sarthe, XVII, 1922, 28-32.

P. Boulanger et alii, La Révolution française 1789-1799 à Confolens, Confolens, 1988.

G. Brejon, « Noms de lieux et d'hommes de la période révolutionnaire ", Bull. de la Soc. des archives hist. de la Saintonge et de l'Aunis, II, 1880, 132-134.

S. Devezeau, Les enfants abandonnés à Angoulême de 1777 à 1815, Thèse, Bordeaux-III, 1983.

J. Giraud, «Les prénoms révolutionnaires à Confolens ", Bull. des Amis du vieux Confolens,

J. Pellisson, « Noms de lieux et d'hommes de la période révolutionnaire ", Bull. de la Soc. des archives hist. de la Saintonge et de l'Aunis, II, 1880, 84-88.

T. Phelipot, « Noms de lieux et d'hommes de la période révolutionnaire », Bull. de la Soc. des archives hist. de la Saintonge et de l'Aunis, II, 1880, 179.

I. Plantivet, Saint-Jean-d'Angély. Étude de démographie historique, 1730-1852, Thèse,

L. de Richemond, « Noms de lieux et d'hommes de la période révolutionnaire », Bull. de la Soc. des archives hist. de la Saintonge et de l'Aunis, II, 1880, 179-180.

J. Seguin, «Influence de la Révolution sur les prénoms des nouveau-nés saintais »,

J. Bourdin, Issoire et les Issoiriens dans la Révolution, Clermont-Ferrand, 1989.

I. Brossard, «Amable, Antoine, Marie et les autres, les prénoms des Riomois au XVIIIesiècle ", L'Histoire en Auvergne, 2, 1995, 153-167. 
P. Chassang, "Actes de baptême rédigés par un prêtre réfractaire, curé clandestin de Vieillespesse (Cantal) », Le Gonfanon, 13, 1985, 11-12.

P.-F. Fournier, «Rectifications d'actes de l'état civil par les administrations du département du Puy-de-Dôme entre 1792 et 1802 ", Mélanges Paul Fournier, Paris, 1929, $249-260$

F. Mège, Un registre d'état civil de l'année 1793, Clermont-Ferrand, 1891.

Docteur de Ribier, "Onomastique révolutionnaire à Saint-Bonnet-de-Salers et à Aurillac », Rev. de la Haute-Auvergne, XXIX, 1935-36, 283-285.

H. Soanen, «Un baptême en 1790 », Annales hist. de la Révolution française, IX, 1932, 164-165.

H. Soanen, "Notes sur la sécularisation de l'état civil dans le district de Thiers", Annales hist. de la Révolution française, XV, 1938, 360-365.

Béarn, $c f$. GascogneBerry

A. Aujeu, «Enfants trouvés à Buzançais (Indre) de 1792 à 1822 », Bull. du groupe d'hist. et d'archéol. du Buzançais, 16, 1984, 69-92.

J. Berducat, « Prénoms au fil du temps. Le choix du prénom », Informations généalogiques, $55,1988,678-684$.

R. Gayon, «Prénoms nouveaux et patronymes malsonnants à Bourges, durant la Révolution ", Cahiers d'archéol. et d'hist. du Berry, 137, 1999, 7-13.

G. Lévêque, "Contribution au bicentenaire: la Révolution des prénoms à Châteauroux ", Cahiers d'Archéol. et d'Hist. du Berry, 104, 1990, 23-31.

R. Marcel, L'abandon et la charité en Berry jusqu'après la Révolution, Bourges, 1990.

Cl. Rioland, "Les prénoms à Poulaines (Indre) à la fin du xviiiesiècle ", Nouvelle Rev. d'Onomastique, 13-14, 1989, 84-100.

Bourbonnais et Nivernais

Aspects de la Révolution dans la Nièvre, Nevers, 1989.

R. Baron, «Notes d'anthroponymie concernant le canton de Varzy aux xviie et au Xviiiesiècles ", Rev. internat. d'onomastique, XXII, 1970, 161-178.

N. Bossut, La déchristianisation dans le district de Corbigny, Maîtrise, Paris-I, s.d.

G. Florenty, «Les prénoms révolutionnaires dans le Val de Loire neversois : un tableau contrasté », Du Nivernais à la Nièvre... Études révolutionnaires, III, Nevers, 1987, 35-59.

J. Gadiou, «À propos de bottes!", Bull. de la Soc. scient. et artist. de Clamecy, XXXVXXXVI, 1911-12, 75-90.

S. Legrand et E. Cocu, «Étude des prénoms révolutionnaires de Cosne-sur-Loire », Du Nivernais à la Nièvre... Études révolutionnaires, V, Nevers, 1989, 131-136.

Docteur Lère, «Un nom de Vichyssois, peu connu : Bourgeois, Capillaire, Républicain », Bull. de la Soc. d'hist. et d'archéol. de Vichy, IV, 1956, 139-141.

E. Liris, La symbolique de la régénération, 1790-1793, Thèse, Paris-I, 1992.

- «Pétition pour obtenir le changement de nom de Louis en celui de Socrate », Aspects de la Révolution dans la Nièvre, Nevers, 1989, 79-80.

Bourgogne 

1989, 53-67.

- "Les prénoms révolutionnaires dans le district de Beaune », Recueil des travaux du Centre beaunois d'études historiques, 11, 1992, 63-74.

- « Les prénoms révolutionnaires en Côte-d'Or », Annales de Bourgogne, 62, 1990, 62-72. sciences hist. et nat. de l'Yonne, 1961-62, 5-16.

- « Les prénoms républicains à Auxerre de 1792 à 1800 », L'Écho d'Auxerre, 81, 1969, 3-8.

- «Les prénoms révolutionnaires à Mirepoix (Ariège) et Montluel (Ain) sous la Révolution », Rev. de Comminges, CVIII, 1993, 73-85.

Bretagne

S. Alcan, Recherches sur la déchristianisation dans le district d'Auxerre, Yonne, Maîtrise, Paris-I, 1976.

E. Blin, « Prénoms auxerrois de l'an II », Annales de Bourgogne, XVI, 1944, 269-270.

P. Bodineau, «Observations sur l'origine des baptêmes civiques dans l'Yonne », Mém. de XXXI, 1972, 209-216.

M. Dorigny, Autun dans la Révolution française, Le Mée-sur-Seine, 1989.

Fr. Kerlouegan, «Les prénoms à Couchey (Côte-d'Or) de 1790 à l'an IX ", Nouvelle Rev. d'onomastique, 13-14, 1989, 101-106.

J. Bazin, «Prénoms révolutionnaires en Bretagne », Cahiers de l'Iroise, 17, 1970, 55.

D. Bernard, «État de naissance de la commune d'Elliant en l'an II, 1793-1794 », Bull. de la Commission diocésaine d'Architecture et d'Archéol. du Diocèse de Quimper et de Léon, 1941, 252.

N. Berthau, Cornélie, Jasmin et Espoir du monde... Les prénoms révolutionnaires en Ille-etVilaine, Maîtrise, Rennes-II, 1995.

A. Croix, «Les prénoms « révolutionnaires » dans les campagnes de Loire-Inférieure », Mélanges Jean Jacquart, Paris, 1994, 155-162.

A. Croix et P. Scilbo (dir.), Brutus, Désir de la Paix et Jonquille. Les prénoms de la Révolution en Loire-Inférieure, Nantes, 1993.

R. Daniel, «Les prénoms révolutionnaires dans le Finistère », Bull. de la Soc. archéol. du Finistère, XCVI, 1970, 173-199.

J. Foucher, "Les prénoms révolutionnaires en Bretagne ", Cahiers de l'Iroise, 16, 1969, 273.

A. Henwood, «Coriandre-Houlette ou Marie-Jeanne, Marat-Finistère ou Jean-Marie. Étude des prénoms à Brest pendant la Révolution (1789-1799) ", Les Cahiers de l'Iroise, 35, 3, 1988, 145-160.

Fr. Lalys, Les prénoms révolutionnaires dans le département du Morbihan, Maîtrise, RennesII, 1992.

C. Le Mercier D'erm, "Prénoms révolutionnaires en Bretagne », Cahiers de l'Iroise, 17, 1970, 109. 
- «Les prénoms révolutionnaires à Dinan: 1789-1799 », Le Pays de Dinan, IX, 1989, 95-103. 337-41. 92. 27. 253-367. 485-487.

G.-M. T., « Prénoms révolutionnaires en Bretagne », Cahiers de l'Iroise, 17, 1970, 55.

A. Trabut, Égalité, Bourrache, Aristide et les autres... Les prénoms révolutionnaires dans le département des Côtes-du-Nord, 1789-1800, Saint-Brieuc, 1993.

Champagne

«Baptême civique à Dun », Bull. de la Soc. des lettres, sciences. et arts de Bar-le-Duc, 1930-33,

J. Berland, «Appellations révolutionnaires et changements de noms de communes de 1790 à l'an VI ", Mém. de la Soc. d'agric., commerce, sciences. et arts de la Marne, LII, 1907-08,

J. Déguilly, «Prénoms et surnoms dans une famille Auboise de 1500 à 1960 », Vie en Champagne, 83, 1960, 8-9.

J.-G. Gigot, «Changements de noms sous la Révolution », Cahiers haut-marnais, 16, 1947,

J. Molin, «Les noms de baptême à Nogent-l'Artaud de 1668 à 1797 », Actes du 103 e Congrès nat. des Soc. savantes (Nancy-Metz, 1978). Section d'hist. moderne, II, Paris, 1979,

J.-P. Monnier, «La révolution dans l'état civil de Saint-Dizier », La Haute-Marne pendant la Révolution, Langres, 1989, 155-165.

Comtat-Venaissin, $c f$. ProvenceComté de Foix, $c f$. GascogneComté de Nice, $c f$. ProvenceDauphiné

R. Chauvet, "Recherches sur les prénoms révolutionnaires dans les Hautes-Alpes », Bull. du Cercle généal. de Midi-Provence, 1, 1989, 3-7.

P. Pavary, La faillite de l'Église constitutionnelle et la déchristianisation dans le département de l'Isère, D.E.S., Grenoble, 1955.

Flandre, $c f$. PicardieFranche-Comté

G. Gazier, "Les prénoms révolutionnaires à Besançon en l'an II", Mém. de la Soc. d'Émulation du Doubs, III, 1923, 127-131.

R.-J. Lovy, Les Cinq Villages des Bois et la Révolution française, Champigny-sur-Marne, 1977.

L. Roy et P. Navand, "Un baptême civique à Lons-le-Saunier en mars 1792 ", Annales hist. de la Révolution française, IX, 1932, 67-69.

J.-M. Thiébaud, "Onomastique révolutionnaire franc-comtoise", Bull. du Centre d'entraide généal. de Franche-Comté, 16, 1983, 5-7.

Gascogne, Béran et Comté de Foix

R. Aymard, Les prénoms sous la Révolution dans les Basses-Pyrénées, Uzos, 1989.

- «Les prénoms dans les Basses-Pyrénées pendant la Révolution", Nouvelle Rev. d'onomastique, 13-14, 1989, 107-130.

Bénétrix, "Les baptêmes républicains dans le Gers ", Rev. de Gascogne, XXXIII, 1892,

M. Darroux, La société populaire de Grenade de 1790 à 1794, D.E.S., Toulouse, 1958.

M. Houth-Baltus, «Les prénoms des habitants de Mirande au xviie et au xviiiesiècles », Rev. internat. d'onomastique, VIII, 1956, 31-42. 
111 J.-P. Jourdan, La Révolution dans les Basses-Pyrénées, Roanne, 1989.

112 J. Labarthié, Étude démographique de Lévignac-sur-Save, 1700-1914, Thèse, Toulouse, 1974.

113 G. Subreville, «Les prénoms révolutionnaires à Mirepoix (Ariège) et Montluel (Ain) sous la Révolution », Rev. de Comminges, CVIII, 1993, 73-85.

114 G. Supéry, « Noms de baptême et prénoms à Lafitte-Vigordane de 1674 à 1914 », Rev. de Comminges, CXCIV, 1981, 445-454.

Guyenne

115 J.-P. Barès, « Les noms et prénoms révolutionnaires à Mur-de-Barrez, 1793-1798 », Rev. du Rouergue, 17, 1989, 163-167.

116 P.-H. Billy, « Les prénoms à Bordeaux pendant la Révolution (1790-an V). Latradition », Nouvelle Rev. d'onomastique, 13-14, 1989, 76-83.

117 J. Donat, "Une application du calendrier républicain aux inscriptions à l'état civil », Mém. de l'Acad. des sciences, inscriptions et belles-lettres de Toulouse, CI, 1933, 83-107.

118 G. Julien, "Le calendrier républicain et son application à Saint-Antonin ", Soc.des Amis du Vieux Saint-Antonin, 1993, 29-36.

119 L. Latour, "Les fêtes révolutionnaires à Villefranche", Bull. de la Soc. des Amis de Villefranche et du Bas-Rouergue, 1989-90, 263-293.

C. Lavergne, Les prénoms à Bordeaux en 1780, 1794, 1819, Maîtrise, BordeauX-III, 1984.

L. Le Cam, "L'état civil dans la commune de Nontron pendant la décennie 1793-1802 », Le Périgord révolutionnaire. Suppl. du Bull. de la Soc. hist. et archéol. du Périgord, 1989, 225-237.

Ph. Loupès, "Les prénominations dans le Bordeaux révolutionnaire ", Rev. française d'Histoire du Livre, 66-67, 1990, 67-82.

M. Lyons, Révolution et Terreur à Toulouse, Toulouse, 1980.

A. Martinaud, «Les prénoms révolutionnaires dans le département du Lot ", Bull. de la Soc. des études litt., scient. et artist. du Lot, CV, 1984, 315-318.

L. Mazars, La Révolution en Rouergue. District d'Aubin. 1789-1795, II, Villefranche, 1978.

- « En pays réolais. Des noms, prénoms et surnoms », Folklore de France, 163, 1979, 21-22.

J. Pontet, «Les prénoms à Saint-Émilion, de la Révolution à la Restauration ", Le prénom. Mode et histoire (Entretiens de Malher 1980), Paris, 1984, 341-354.

- «La Révolution de 1789 et les prénoms », Amitiés généal. bordelaises, 30, 1990, 19-20.

E. Sol, L'ancien état civil de Quercy, Paris, 1924.

Île-de-France

R. Bange, "Recherches sur les prénoms révolutionnaires à Paris », Annales hist. de la Révolution française, 1994, 39-65.

J. Bernet, « L’iconoclasme verbal sous la Révolution française (1792-1795). Changements de toponymes, patronymes, prénoms... dans le district de Compiègne ", Annales hist. compiégnoises modernes et contemporaines, 7, 1979, 33-44.

- «Les prénoms républicains sous la Révolution française l'exemple du district de Compiègne, 1793-1795 », Le prénom. Mode et histoire (Entretiens de Malher 1980), Paris, 1984, 247-253.

-Recherche sur la déchristianisation dans le district de Compiègne, Thèse, Paris-I, 1980. 
S. Bianchi, « Liberté ou Marie. Enquête sur 8000 prénoms », 89 en Essonne, 6, 1990, 66-70.

- «Manifestations et formes de la déchristianisation dans le district de Corbeil », Rev. d'histoire moderne et contemporaine, XXVII, 1979, 256-281.

- «Les prénoms "révolutionnaires" dans la Révolution française », La Révolution et l'ordre juridique privé. Rationalité ou scandale? (Actes du Colloque d'Orléans, 11-13 sept. 1986), Paris, 1988, 109-124.

- «Les prénoms « révolutionnaires » en l'an II l'exemple de Corbeil et de la Seine-etMarne », Le prénom. Mode et histoire (Entretiens de Malher 1980), Paris, 1984, 255-270.

131 J. Blécon, "Les prénoms "révolutionnaires " à Rambouillet », Bull. de la Soc. hist. et archéol. de Rambouillet et de l'Yveline, 55-56, 1993, 5-12.

R. Chatel, Recherches sur la déchristianisation dans le district d'Étampes, Maîtrise, Paris-I, 1979.

133 Ph. Daumas, Familles en Révolution (1785-1815), D.E.A., Rennes-II, 1993.

- "Familles et mentalités en Ile-de-France (1775-1825), une révolution différée ", Colloque La culture paysanne (Rennes, 1993), in Annales de Bretagne et des pays de l'Ouest, 100, 1993, 411-423.

134 M. Dommanget, "Débaptisation collective de prénoms ", Annales hist. de la Révolution française, 1952, 326-328.

- «La déchristianisation à Beauvais. Les laïcisations de noms et de prénoms ", Annales révolutionnaires, 8, 1916, 230-249.

135 J. Dufour, La vie religieuse et la déchristianisation dans le district de Saint-Germain-en-Laye pendant la Révolution française, Maîtrise, Paris-I, 1984.

136 J. Dupâquier, «Prénoms, parrains, parenté. Recherche sur les familles du Vexin français de 1540 à 1900 », Mém. de la Soc. hist. et archéol. de Pontoise, du Val-d'Oise et du Vexin, LXIX, 1980, 55-87.

137 M. Garnier, «Les prénoms révolutionnaires donnés aux enfants de Vincennes (1792-1796)», Clio 94, 8, 1989, 242, 287.

- «Recherche des prénoms révolutionnaires donnés aux enfants nés à Vincennes de 1792 à 1796 », Vincennes Histoire, 2, 1989, 23-27.

P. Gauthier, Vivre, se marier, mourir à Melun sous la Révolution française (1789-1799), D.E.A., Paris-I, 1988.

139 M. Guillot, «Une application du calendrier républicain. Les prénoms de l'an II dans le canton de Nanterre ", Paris et Ile-de-France. Mém. publiés par la Fédér. des Soc. hist. et archéol. (6e Colloque, avril 1989), LI, 1990, 203-221.

B. Hauviller, «La vie quotidienne à Bourron-Marlotte sous la Révolution et l'Empire : mémoires d'un état civil », Les Amis de Bourron-Marlotte, 23, 1989, 8-13.

M.-Th. Larroque, «Liberté ou Marie. Prénoms de la ville, prénoms des champs », 89 en Essonne, 6, 1990, 57-65.

142 E. Lévy, «Les prénoms parisiens de l'an II », Révolution française, LXXIII, 1920, 134-142, 225-247.

143 C. Maillier, Les rues de Dreux, Dreux, 1980.

- «Un peu d'histoire. Les prénoms républicains », Bull. municipal officiel de BoulogneBillancourt, 96, 1959, 3.

144 L. Plancouard, Étude sur les prénoms en usage dans le Vexin, Pontoise, s.d. 

mentalités, spiritualités (Colloque de Chantilly, 1986), Turnhout, 1988, 300-308.

- "Le test des prénoms et la période révolutionnaire», Bull. du Centre d'hist. contemporaine du Languedoc méditerranéen-Roussillon, 40, 1987, 28-31. 271-275. 
164 G. Floutard, «À travers les registres d'état civil», Lettre des Amis des archives départementales de Haute-Garonne, 49, 1988, 2-4.

P. Lashermes, G. Joubert, «Quelques surnoms révolutionnaires », Bull. hist., scient., litt., artist. et agricole publié par la Soc. acad. du Puy et de la Haute-Loire, 1989, nº́spécial, 127-128.

R. Laurent et G. Gavignaud, La Révolution française dans le Languedoc méditerranéen, Toulouse, 1987.

R. N., « La Révolution française et les prénoms », Folklore de l'Aude, II, 1939, 286-293.

168

M. Paculi, « Les prénoms de la Révolution en Agde », Cercle généal. de Languedoc, 25, 1984 , 13-14.

J.-M. Piques, Démographie et subsistances à Bédarieux, 1787-1801, D.E.S., Montpellier, 1967.

170 M. de Poitevin, «Les prénoms révolutionnaires à Albi et à Castres ", Soc. des sciences, arts et belles-lettres du Tarn, XLII, 1988 (1990), 705-716.

171 G. Valantin, «Fantaisies révolutionnaires », Bull. hist., scient., litt., artist. et agricole publié par la Soc. acad. du Puy et de la Haute-Loire, LIV, 1978, 143.

Limousin et Marche

172 N. Bertrand, «La mode des prénoms du xviie au xixe dans un village creusois », Ethnologia, 6-7, 1978, 117-121.

173 J. Boulaud, «Le Livre de raison de Grégoire Benoist de Lostende, trésorier de France au bureau des Finances de Limoges (1677-1754) », Bull. de la Soc. archéol. et hist. du Limousin, LIX, 1909, 75-129.

174 L. Jouhaud, "Prénoms révolutionnaires ", Bull. de la Soc. archéol. et hist. du Limousin, LXXIX, 1941-42, 146-149.

J.-F. Pérol, «Le "fricotage" de "Verge d'or-caille-lait" avec "Belle de nuit-sans culote !" ", Lemouzi, 74, 1980, 125-140.

L. Perouas et alii, Léonard, Marie, Jean et les autres. Les prénoms en Limousin depuis un millénaire, Paris, 1984.

177 J. Teyssandier, Étude démographique, économique et sociale de Collonges-la-Rouge (Corrèze) de 1759 à 1800, Maîtrise, Toulouse, 1970.

178 D. Weingarten, La tentative de déchristianisation a-t-elle réussi en Corrèze Essai ou aperçu sur la vie religieuse des populations corréziennes (1795-1810), Maîtrise, Limoges, 1984.

Lorraine

179 « Baptême civique à Dun », Bull. de la Soc. des lettres, sciences et arts de Bar-le-Duc, 1930-33, 92.

180 J.-J. Barbé, « Les prénoms de l'an II à Metz », Les Amis de Metz, 5, 1962, 5-9.

- «Les Prénoms révolutionnaires, 1793-1794 », Confrérie de Marie-Immaculée. Cuuvre des vocations du Diocèse de Metz, 1951, 16-18.

181 E. Duvernoy, «Nom révolutionnaire de Villey-Saint-Étienne », Bull. mensuel de la Soc. d'archéol. lorraine et du musée lorrain, I, 1901, 186-187.

E. Mangenot, Les registres paroissiaux de Nancy pendant la Révolution française, Nancy, 1897.

M. Sary, « Les prénoms de l'an II à Metz », Vivre à Metz, janv. 1982, 26-27.

184 J. Thouvenot, "Les enfants trouvés à Nancy (1793-1830) ", Actes du 75e Congrès nat. des Soc. savantes (Nancy, 1950). Section d'hist. moderne, Paris, 1951, 101-121.

Lyonnais 
R. Bange, La déchristianisation dans le district de Villefranche-sur-Saône pendant la Révolution française l'exemple des prénoms, Maîtrise, Lyon-II, 1990.

- "Révolution et mentalités: les prénoms à Villefranche-sur-Saône, capitale du Beaujolais », Actes du Colloque international «Ville et Révolution française » (Lyon, mars1993), Lyon, 1994, 273-286.

186 J. Barou, «Les enfants abandonnés à Montbrison de la fin de l'Ancien Régime à l'Empire (1773-1815) », Bull. de la Diana, LI, 1989, 85-113, 149-187.

187 M.-Cl. Bonnefond, La déchristianisation dans le district de Roanne à travers l'exemple des prénoms révolutionnaires, 1789-1799, Maîtrise, Lyon-II, 1992.

B. Cousin, La déchristianisation à Lyon l'exemple des prénoms révolutionnaires, 1789-1799, Maîtrise, Lyon-II, 1991.

189 J.-B. Galley, Saint-Étienne et son district pendant la Révolution, II, Saint-Étienne, 1904.

190 E. Lévy, « Les Prénoms de l’an II dans la région lyonnaise », La Municipalité française, 15 août 1921,1 et 67.

191 G. Liogier, "L'état civil stéphanois sous la Terreur », Bull. du Vieux Saint-Étienne, 156, 1989, 79-82.

S. Maury, La déchristianisation dans le district du Lyonnais l'exemple des prénoms révolutionnaires, Maîtrise, Lyon-II, 1990.

Maine, $c f$. AnjouMarche, $c f$. LimousinNivernais, $c f$. BourbonnaisNormandie

M. Baudot, «Les prénoms de l'époque révolutionnaire dans le département de l'Eure », Rev. hist. de droit français, 1934, 749-750.

M. Chevreuil, « Prénoms révolutionnaires », Pays d'Argentan, IV , 1932, 15-16.

L. Denis, "Notes sur les enfants trouvés à Rouen", Rev. des Soc. savantes de HauteNormandie, 42, 1966, Lettres, 35-42.

196 A. Dupont, « En marge de la « révolution culturelle » de l'an II. Fantaisies toponymiques et anthroponymiques ", Rev. du département de la Manche, 32, 1990, 45-70.

- "Les prénoms révolutionnaires dans l'arrondissement de Cherbourg », Le Viquet, 82, 1988, 53-68.

- «Prénoms révolutionnaires dans le Centre-Manche », Le Viquet, 86, 1989, 62-75.

C. Féron, «Sous la Révolution. L'influence de la Terreur sur les prénoms donnés aux enfants dieppois en 1793 et en 1794 », Les Amys du Vieux Dieppe, XCIV, 1987, 8-11.

Ph. Goujard, "Le stock de prénoms en Pays de Caux, 1686-1795 ", Le prénom. Mode et histoire (Entretiens de Malher 1980), Paris, 1984, 203-207.

199 A. Hauchecorne, "Un épisode envermeudois de la Révolution française le baptême civique d'un officier municipal, 20 novembre 1793 », Connaissance de Dieppe, 49, 1988, $1-2$.

200 O. Jouault, «Le prénom et la Révolution à Avranches (1789-1799) », Rev. du département de la Manche, 32, 1990, 71-77.

201 F. Lechanteur, "Les prénoms à Agon (Manche), pendant trois siècles ", Mélanges Karl Michaëlsson, Göteborg, 1952, 295-310.

202 M. Le Pesant, « Prénoms révolutionnaires et contre-révolutionnaires dans l'Eure », Bull. de la Soc. des antiquaires de Normandie, LVIII, 1965-68, 482-489. 

1660-1850, Thèse, Paris-I, 1993. 1989, 413-436. Manche, XXVI, 1984, 71-80. 1789-1800, Rouen, 1992, 145-150. Viquet, 89, 1990, 35-38. picarde, 5, 1962, 28-31.

Orléanais

P. Daire, « Des prénoms révolutionnaires », Gâtinais généal., 3, 1994, 9. Montargis, 17, 1983, 21-22.

A. Magnier, Religion et Révolution en Eure-et-Loir, Maîtrise, Paris-I, 1986. Mézilles (Yonne) », Mélanges Jacques Dupâquier, Paris, 1993, 435-448. hist. et nat. de l'Yonne, 122, 1990, 103-114.

Picardie, Artois et Flandre en Cambrésis, 49, 1991, 43-46. 515-516. Linguistique picarde, 24, 1967, 20-32. VIII, 1980-82, 144. XX, 1898-1900, 445-454. 47-61. 356.

J.-P. Lethuillier, Idéologie et mentalités l'essor de l'individualisme en Basse-Normandie,

- "Prénoms et Révolution: enquête sur le corpus falaisien ", Annales de Normandie, 4,

P. Manneville, «Les prénoms révolutionnaires au Havre», Rev. du département de la

M.-F. Niel, "Louviers et la Révolution », A travers la Haute-Normandie en Révolution,

J. Pierre et A. Dupont, « Les prénoms révolutionnaires à Avranches de 1793 à 1803 », Le

J. Seguin, «En feuilletant de Vieux Registres », Rev. de l'Avranchin, XXVIII, 1935, 218-221.

J. Vacandard, «Prénoms rares ou curieux de la commune de Melleville», Linguistique

J. Jourdain, "En feuilletant le registre des naissances de l'an II", Amis du Vieux

G.Leloup, "Calendrier républicain et état civil à Montargis pendant la Révolution ", Bull. de la Soc. d'Émulation de l'arrondissement de Montargis, 69, 1985, 224-232.

J.-P. Pélissier, « Les Romarins ancêtres des Jules. Les prénoms agricoles des ans II et III à

R. et S. Pélissier, «Les prénoms révolutionnaires à Mézilles ", Bull. de la Soc. des sciences

M.-L. Reneaud, «Pour ne pas quitter Griselles. Prénoms révolutionnaires à Griselles », Bull. de la Soc. d'Émulation de l'Arrondissement de Montargis, 71, 1986, 66-67.

S. Besin, «Quand les petits Cambrésiens s'appelaient Brutus ou pomme de terre », Jadis

M. Chartier, «Un prénom original », Annales hist. de la Révolution française, XXIV, 1952,

M. Cury, G. Railliet, "Folklore d'Archon et environs. Du berceau à la tombe ",

P. Delattre, «Les enfants trouvés à Douai sous la Révolution et l'Empire », Amis de Douai,

A. Dubois, « Les noms de baptême à Amiens ", Bull. de la Soc. des antiquaires de Picardie,

W. Éloy, «A travers les registres de l'état civil. Choix des prénoms en Picardie, pendant la période révolutionnaire (an II et an III : 1793-1794) », Linguistique picarde, 19-20, 1966,

L. Jacob, « Un baptême civique en 1791 », Annales hist. de la Révolution française, IX, 1932,

B. Lefebvre, Douai sous la Révolution, 1789-1799. Étude démographique, Douai, 1975. 

1981, 119-141. Cambrésis, 15, 1982, 9-10. Haveluy au XviIIe siècle, Paris, 1997. Linguistique picarde, 16, 1965, 2-14. Calaisis, 116/117, 1988-89, 277-295. départementale d'hist. et d'archéol., XII, 4, 1989, 395-398. de l'an III) ", Bull. hist. et artist. du Calaisis, 86, 1981, 145-153.

Poitou $\mathrm{XV}, 1982,63$. $12-16 ; 43,1969,11-14$. 1952.

Provence, Comtat Venaissin et Comté de Nice (1789-an VIII), Millau, 1981. 1570-1870), Maîtrise, Aix-I, s.d. hist. et géogr., IV, 1933-36, 525-529. 1991, 123-174. Bull. de la Soc. des Amis du Vieux Toulon, 1924, 39-51. Draguignan, XXXIV, 1922-23, 72-82.

R. Legrand, «Prénoms révolutionnaires », Bull. de la Soc. d'Émulation d'Abbeville, XXV, 1 ,

F. Machelart, "Surnoms donnés aux enfants trouvés à Cambrai en 1793 ", Jadis en

G. Tassin, Anthroponymie et changement dans une société villageoise. Les noms de personne à

G. Vasseur, "Les noms de baptême et les prénoms de Nibas (Somme), 1599-1959 ",

A. Vion, «Les prénoms républicains dans le district de Calais », Bull. hist. et artist. du

- "Les prénoms républicains dans le Pas-de-Calais», Bull. de la Commission

A. et R. Vion, « La déchristianisation des prénoms en Calaisis (de novembre 1792 à la fin

P. Arches, «Les prénoms à Niort pendant la Révolution: 1789-1799», Actes du 108e Congr. nat. des Soc. savantes (Grenoble, 1983). Section d'Hist. moderne, II, Paris, 1984, 127-147. - «Les prénoms révolutionnaires à Gourgé », Bull. de la Soc. hist. et scient. des Deux-Sèvres,

P. Durand-Epaud, «Olonne en 1793 », Bull. de la Soc. Olona, 39, 1965, 9-16; 40, 1966,

G. Fradet, «Les prénoms révolutionnaires à Poitiers », Histoire et Généal., 34, 1991, 27-37.

J. Marcade, "Châtellerault à la fin du XVIIIe siècle », 111e Congrès nat. des Soc. savantes (Poitiers, 1986). Section d'hist. moderne et contemporaine, II, Paris, 1986, 23-38.

4 J. Perret, Histoire de la Révolution française en Poitou-Charentes, 1789-1794, Poitiers, 1988.

Marquis H. de Roux, Histoire religieuse de la Révolution à Poitiers et dans la Vienne, Lyon,

C. Derobert-Ratel, Institutions et vie municipale à Aix-en-Provence sous la Révolution

P. Échalier, Prénommer en Provence de la fin du xvIe siècle à la fin du XIXe siècle (Arles,

L. Honoré, «Au sujet d'un curieux prénom révolutionnaire à Solliès en 1793 », Le Var

D. Maure, «Jean-Jacques Rousseau et Guillaume Tell nés à Marseille en 93 le hochet à l'épreuve", Tout le portrait de son père. Des paroisses à l'état civil. Marseille, 1586-1889. Répertoire des séries GG et E. Étude sur les prénoms révolutionnaires marseillais, Marseille,

A.-J. Parès, « Les Curiosités de l'état civil à Toulon pendant la Révolution (1792-1802) »,

- «Les prénoms révolutionnaires à Toulon», Bull. de la Soc. d'études scient. et archéol. de 
241 M.-J. Rembado, Prénoms en temps long en Provence, Brignolles 1580-1880, Maîtrise, Aix-I, 1984.

P. Roux, «A propos des prénoms dans le Var à l'époque révolutionnaire », Annales du Sud-est varois, 14, 1989, 91-98.

- «Prénoms révolutionnaires : quelques lueurs sur les prénoms en Provence, à l'époque révolutionnaire », Bull. de l'acad. du Var, nº spécial, 1989, 187-203.

L.-P. Sardella, La déchristianisation dans le quart sud-est de la France. Essai de cartographie historique, Maîtrise, Aix-I, 1971.

244 M. Villard, «Du sacrement à l'état civil une mutation sans révolution », La Gazette des Archives, 146-147, 1989, 290-300.

- «Prénoms révolutionnaires à Toulon », Bull. de l'acad. du Var, nº spécial, 1989, 204-208.

Roussillon

A. Chuquet, Le général Dagobert (1736-1794), Paris, 1913.

R. Cobb, «Débaptisations à Perpignan ", Annales hist. de la Révolution française, CXXXVII, 1954, 361-362.

247 J. Guibeaud, «Les naissances hors mariage à Perpignan de 1684 à 1894 », Soc. agric., scient. et litt. des Pyrénées-Orientales, XXVI, 1895, 431-446.

Saintonge, $c f$. AunisSavoie

Cl. Grange, Étude de la déchristianisation dans le district de Moutiers (Savoie) durant l'an II, Maîtrise, Paris-I, 1979.

G. Letonnelier, « Sur les prénoms de l'an II », La Rev. savoisienne, LV, 1914, 11-12.

Touraine, $c f$. Anjou 